\title{
Mouse duodenum as a model of inflammation induced by enterotoxigenic Escherichia coli K88
}

\author{
Kai Wang, Yu Qi, Shushuai Yi, Zhihua Pei, \\ $\mathrm{Na}$ Pan, Guixue $\mathrm{Hu}$ \\ College of Animal Science and Technology, \\ Jilin Agricultural University, Xincheng, Changchun 130118, People's Republic of China \\ guixue1964@126.com
}

Received: October 24, 2015 Accepted: February 25, 2016

\begin{abstract}
Introduction: The aim of the experiment was to establish the enterotoxigenic Escherichia coli K88 (ETEC K88)-induced $\mathrm{BALB} / \mathrm{c}$ mouse duodenum inflammation model. Material and Methods: Mice were administered different concentrations of E. coli $\mathrm{K} 88\left(1.0 \times 10^{7}-10^{9} \mathrm{CFU} / \mathrm{mL}\right)$ for $3 \mathrm{~d}$ by means of an esophageal catheter. Results: The results showed that the treated group expressed several significant clinical symptoms, such as reduced dietary demands and weight loss, an increased presence of IL-1 $\alpha$, TNF- $\alpha$, and MPO in the peripheral blood, and some pathological changes in the duodenum. On the $6^{\text {th }}-8^{\text {th }}$ days, the body weight of the mice was the lowest. On the $8^{\text {th }}$ day, there were significant differences in IL- $1 \alpha$, TNF- $\alpha$, and MPO levels compared to the control group $(\mathrm{P}<0.05)$, the gap between the duodenum mucous layer and the muscular layer had widened, the number of goblet cells was increased, and the inflammatory infiltrate and inflammation changes in the lamina propria and the mucous layer were the most obvious. Conclusion: The duodenum inflammation was the most severe on day 8; thus, the model was successfully established. In addition, varying concentrations of ETEC K88 did not significantly influence the duodenum inflammation $(\mathrm{P}>0.05)$.
\end{abstract}

Keywords: mouse, duodenum, enterotoxigenic Escherichia coli, inflammation model.

\section{Introduction}

Intestinal inflammation is generally divided into the following groups based on its different causes: bacterial-, viral-, and antibiotic-associated inflammatory bowel diseases (IBD). To understand the mechanisms of the diseases, some experimental models have been created $(1,5,6)$. Animal models of intestinal inflammation have provided a wealth of information about mucosal immunology, particularly as it relates to the maintenance of intestinal homeostasis, the disruption of homeostasis, and the intestinal inflammation encountered in IBD (13). In the last 50 years, more than 60 distinct animal models of IBD have been developed, and approximately 40 of these are currently used in experimental protocols (8). Piglet diarrhoea is an infectious disease with high incidence and mortality, mainly caused by enterotoxigenic Escherichia coli K88 (ETEC K88), and it can cause severe economic losses in the pig industry (3). The treatment of the disease primarily depends on antibiotics. However, long-term use of some antibiotics will result in drug residues, bacterial resistance, immune suppression, and environmental pollution. Probiotics can enhance immune function and have a therapeutic effect on animal intestinal inflammation. To study the mechanism of probiotics in treating intestinal inflammation, the establishment of an ETEC K88-infected animal duodenum inflammation model is a necessary prerequisite for the experiment. Mice, the most commonly used laboratory animals, have phylogenetic similarities and are easy to handle, making them the preferred choice for establishing animal models. In this study, an experimental model of duodenum inflammation due to infection with ETEC K88 was established, which will provide a foundation for studying the immune-boosting mechanism of probiotics on animal intestinal inflammation. 


\section{Material and Methods}

Animals. SPF BALB/c male mice were purchased from Beijing HFK Bioscience (China). The mice were housed in polystyrene cages with stainless steel wire lids and given water and chow ad libitum. The housing was maintained at a constant temperature $\left(21-22^{\circ} \mathrm{C}\right)$ with a 12-h light-dark cycle. The mice were of 4-6 weeks of age. The surviving mice were humanely sacrificed using cervical dislocation, abiding by the laboratory animal management regulations of China.

E. coli culture. ETEC K88 C83912 was provided by the Institute of Microbiology, College of Animal Science and Technology, Jilin Agricultural University. The culture, prepared as previously described (10), contained approximately $10^{9}$ colony-forming units (CFU) per millilitre. The bacteria were washed in PBS, centrifuged (2000 r/min, $10 \mathrm{~min}$ ), and the sediment was re-suspended to prepare a final inoculum of $10^{8}$ and $10^{7} \mathrm{CFU} / \mathrm{mL}$ ETEC K88 in PBS.

Experimental design. Mice numbering 72 individuals were randomly divided into four groups: A, $\mathrm{B}, \mathrm{C}$, and a control group. The mice were pretreated with $0.2 \mathrm{~mL}$ of $1 \% \mathrm{NaHCO}_{3}$. After $30 \mathrm{~min}$, group A was administered $1.0 \times 10^{7} \mathrm{CFU} / \mathrm{mL}$, group B $1.0 \times 10^{8}$ $\mathrm{CFU} / \mathrm{mL}$, and group $\mathrm{C} 1.0 \times 10^{9} \mathrm{CFU} / \mathrm{mL}$ of ETEC $\mathrm{K} 88$, intragastrically, by means of an esophageal catheter, and the control group was administered the same dose of normal saline. The mice were treated once a day for $3 \mathrm{~d}$ with $0.2 \mathrm{~mL}$ of $1 \% \mathrm{NaHCO}_{3}$ and ETEC K88 per mouse. The well-being status, faecal characteristics, and activity of the mice were observed every $6 \mathrm{~h}$. The intestinal contents of the mice with inflammation were discarded, and the gut cavity was repeatedly washed with PBS. The washing fluid was collected for isolation, identification, and pathogenicity testing for E. coli. Simultaneously, the bacterial gene sequence of 16S rRNA was determined by PCR (4).

Disease activity index (DAI). The body weight of the mice was recorded twice a day using the Cooper method and then averaged (14). Double-blind scoring was conducted regarding enteritis according to observations of fresh mice faeces (Table 1).

Histopathology. The entire duodenum was excised and cleaned with normal saline, fixed in $10 \%$ neutral buffered formalin, and embedded in paraffin. Next, $4 \mu \mathrm{m}$-thick longitudinal sections were cut and stained with haematoxylin and eosin (H\&E).
Determination of myeloperoxidase (MPO), TNF- $\boldsymbol{\alpha}$, and IL-1 $\boldsymbol{\alpha}$. To validate the inflammatory changes in the duodenum, orbital venous blood samples of three mice from each group were collected on days $0,4,6,8,10$, and 12, centrifuged, and the levels of MPO, TNF- $\alpha$, and IL- $1 \alpha$ were determined with an ELISA kit (Lengton Bioscience Co., China), according to the manufacturer's instructions.

Statistical analyses. The results are presented as the means \pm the standard error of the means (SEM). The statistical analysis was performed using SPSS 18.0 software (IBM, USA). The inflammatory cytokine levels (IL- $1 \alpha$, TNF- $\alpha$, and MPO) for each time point were subjected to one-way analysis of variance (ANOVA), and multiple comparison tests (Tukey test) were used to determine the significance level between groups. Bacterial concentrations and time were analysed using ANOVA and Tukey test. The results were considered significant at the $95 \%$ level of confidence $(\mathrm{P}<0.05)$.

\section{Results}

Clinical symptoms. Before the beginning of the experiment, there was no significant difference in body weight between the different groups $(\mathrm{P}>0.05)$, and the stools were granular. The experimental groups (A, B, and $C$ ) were administered different concentrations of ETEC K88 for $3 \mathrm{~d}$, and the mice in each of the experimental groups appeared depressed and had reduced feed intake. Additionally, other symptoms such as loose stools and occult faecal blood were observed in some mice. On the $6^{\text {th }}-8^{\text {th }}$ days, the body weight of the mice was reduced to its lowest point (Fig. 1).

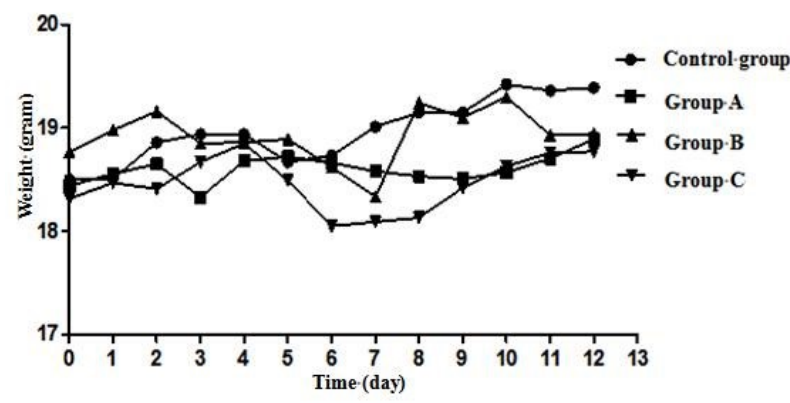

Fig. 1. Changes in body weight of the mice

Table 1. Disease activity index scores

\begin{tabular}{|c|c|c|c|c|c|}
\hline Scores & 0 & 1 & 2 & 3 & 4 \\
\hline Loss of weight & Normal & $1 \%-5 \%$ & $5 \%-10 \%$ & $10 \%-20 \%$ & $>20 \%$ \\
\hline Stool consistency & Normal & & & Loose stools & Watery diarrhoea \\
\hline Occult blood in faeces & Negative & & & Positive & Bloody stools \\
\hline
\end{tabular}

$\mathrm{DAI}=($ weight loss scores + stool viscosity scores + haematochezia scores $) / 3$

Normal faeces: granular stool; loose stool: mushy stool that did not stick to the anus; diarrhoea: watery stool that stuck around the anus 
DAI scoring of ETEC K88-induced duodenum inflammation. In the model groups, the mice produced loose stools and demonstrated weight loss on the $4^{\text {th }}$ day, and the average DAI score was $0.264 \pm 0.3346$. Loose stools and weight loss were observed in nine mice on the $5^{\text {th }}$ day, occult blood was found in seven mice on the $6^{\text {th }}$ day, and all of the mice had occult blood on the $7^{\text {th }}$ day, when the average DAI score was $1.913 \pm 0.2532$. After the $8^{\text {th }}$ day, the DAI scores began to decline. Groups A, B, and C demonstrated similar parameters as regards stool shape, occult blood, and weight loss. On the $6^{\text {th }}-8^{\text {th }}$ days, all mice produced loose stools, and there were no significant differences between groups $\mathrm{A}, \mathrm{B}$, or $\mathrm{C}(\mathrm{P}>0.05)$. The DAI scores of the control group were 0 (Fig. 2).

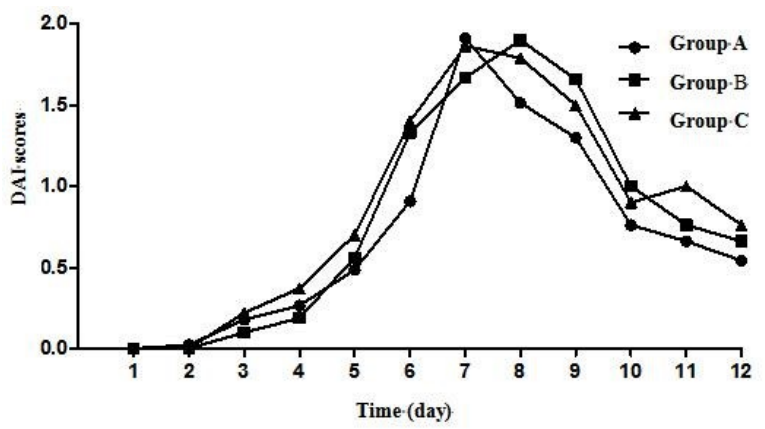

Fig. 2. Disease activity index of the mice $*-\mathrm{P}<0.05$

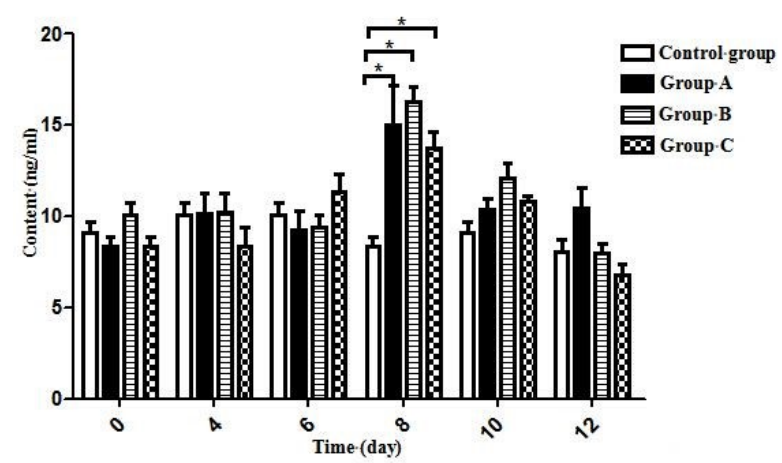

Fig. 3. Changes of MPO in the peripheral blood of the mice $*$ - $\mathrm{P}<0.05$

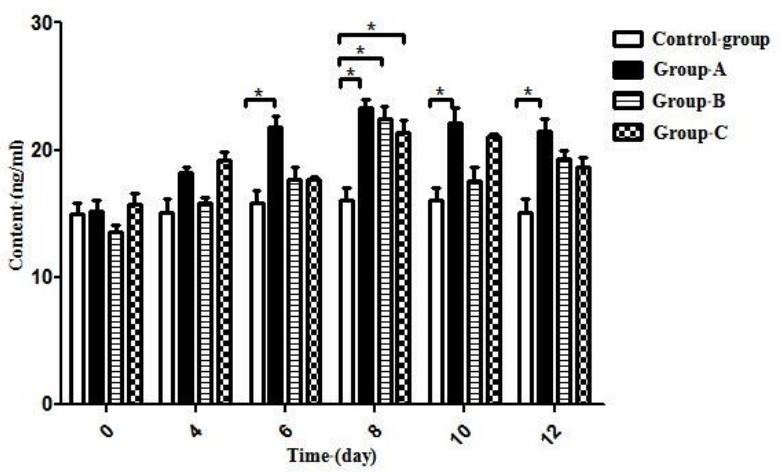

Fig. 4. Changes of TNF- $\alpha$ in the peripheral blood of the mice * $-\mathrm{P}<0.05$

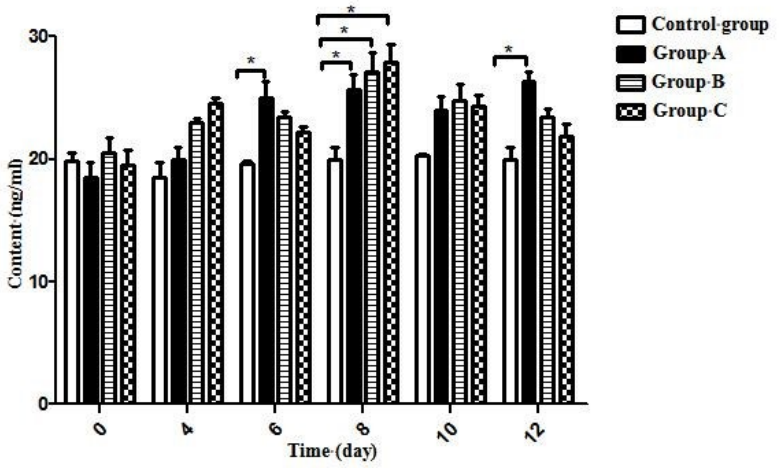

Fig. 5. Changes of IL-1 $\alpha$ in the peripheral blood of the mice * $-\mathrm{P}<0.05$

MPO, TNF- $\alpha$, and IL-1a levels in the peripheral blood. There were no significant differences in MPO, TNF- $\alpha$, or IL- $1 \alpha$ levels in the peripheral blood $(\mathrm{P}>0.05)$ after administration of different concentrations of ETEC K88 $\left(1 \times 10^{7}\right.$ $\left.\mathrm{CFU} / \mathrm{ml}-1 \times 10^{9} \mathrm{CFU} / \mathrm{mL}\right)$ on different days (Figs 3-5).

However, the levels of MPO, TNF- $\alpha$, and IL- $1 \alpha$ among groups $\mathrm{A}, \mathrm{B}$, and $\mathrm{C}$ were significantly different compared to the levels observed in the control group on day $8(\mathrm{P}<0.05)$ (Fig. 6).

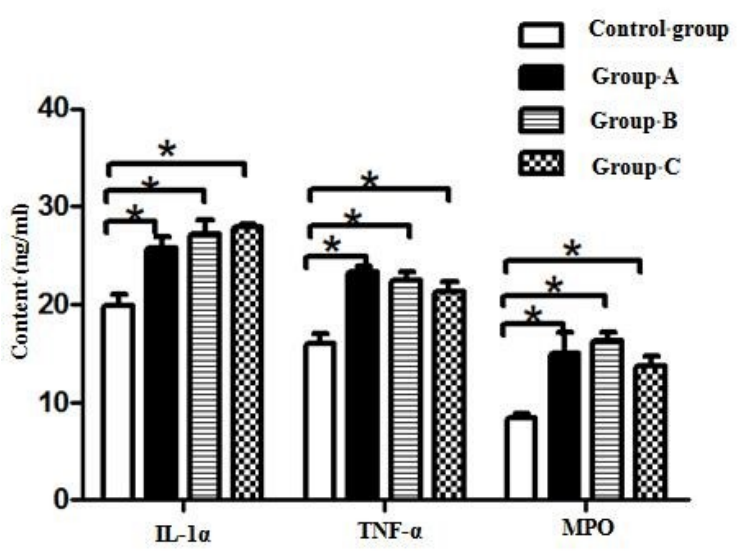

Fig. 6. Changes of IL-1 $\alpha$, TNF- $\alpha$ and MPO in the peripheral blood of the mice on day 8 $*_{\text {- }} \mathrm{P}<0.05$

Histopathological lesions in the duodenum. There were no significant pathological changes in the duodenum of the mice in the control group (Fig. 7A). The pathological lesions in groups $\mathrm{A}, \mathrm{B}$, and $\mathrm{C}$ were similar. On the $4^{\text {th }}$ day, there was a small quantity of inflammatory secretions at the edge of the intestinal villi, but other obvious pathological changes were not observed. However, on the $6^{\text {th }}$ day, the epithelial cells of the duodenal villi were detached, the mid-gut glands in the lamina propria were proliferated, the spatium among intestinal glands was widened, the number of goblet cells was increased, and the infiltration of inflammatory cells, such as lymphocytes and neutrophils, was observed in the lamina propria and mucous layers. On the $8^{\text {th }}$ day, the pathological changes were most severe: the intestinal villi were disintegrated, 
a large number of goblet cells were observed in the mucous layer, the enteric cavity was filled with inflammatory secretions, the spatium among intestinal glands was widened, and a large amount of tissue fluid was exuded from the spatium, forming the oedema. After the $12^{\text {th }}$ day, the condition of inflammation was improved and the number of goblet and inflammatory cells was reduced (Figs 7 B, C, and D).

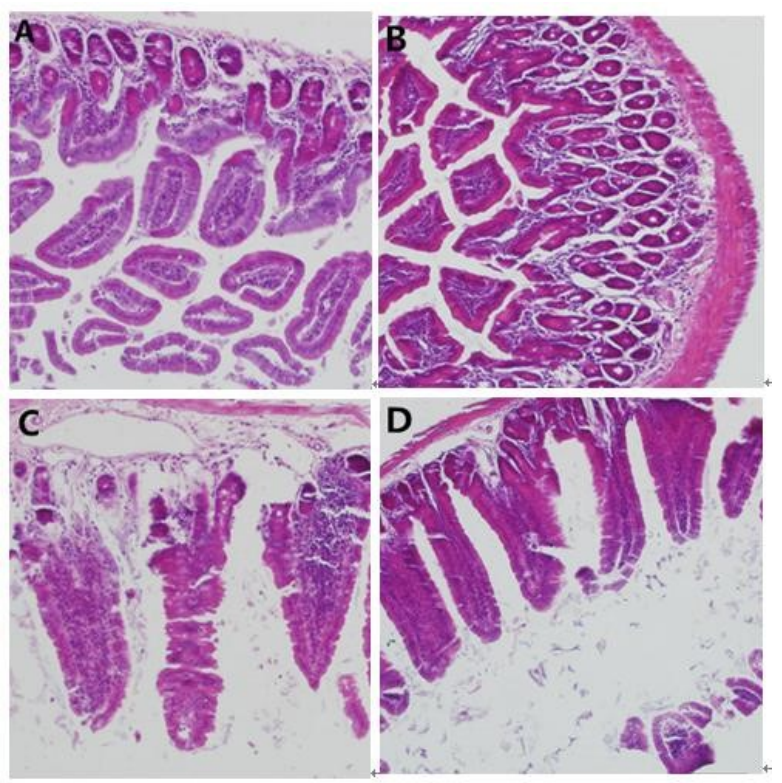

Fig. 7. Histopathology of the intestinal mucosa on various days $\mathrm{H} \& \mathrm{E}$. A - control group; B - changes in model groups on day 4; C - changes in model groups on day $8 ; \mathrm{D}$ - changes in model groups on day 12

\section{Discussion}

It has been reported that Escherichia coli concentrations for the enteritis model have been established at $10^{7} \mathrm{CFU} / \mathrm{mL}$ (7), $1.0 \times 10^{8} \mathrm{CFU} / \mathrm{mL}$ (14), and $1.0 \times 10^{9} \mathrm{CFU} / \mathrm{mL}$ (11). To study the effects of different bacterial concentrations on the modelling time and inflammation degree, the same concentrations of ETEC K88 were used in this experiment. The results showed that the differences in the inflammation time and the secretory volume of inflammatory cytokines between the different bacterial concentrations were not significant $(\mathrm{P}>0.5)$. To determine whether intestinal inflammation was induced by ETEC K88, the intestinal contents were discarded, and the gut cavity was repeatedly washed with PBS. The washing fluid was collected for isolation, identification, and pathogenicity testing for $E$. coli. Simultaneously, the bacterial gene sequence of 16S rRNA was determined by PCR. All of these results proved that ETEC K88 C83912 could colonise the intestines and induce duodenum inflammation.

$\mathrm{BALB} / \mathrm{c}$ mice are the most common laboratory animals. Their price is low and feeding and management is easy, so they are useful for the establishment of ETEC K88-induced duodenum inflammation models. ETEC K88 does not only disturb the functions of absorption and secretion of intestinal epithelial cells, but also induces extensive and obvious inflammation with frequent oedema and cellular degeneration in the small intestine (2). In recent years, some E. coli-induced animal models have been reported. Most of them were induced by intraperitoneal injection and a nasal feeding method. However, the major pathway of E. coli infection is the digestive tract. In this study, we used intragastric administration to induce the duodenum inflammation. In order to reduce the gastrointestinal barrier function and ensure the survival and multiplication of the ETEC K88, $1 \%$ sodium bicarbonate solution was given by gavage. However, the mechanism of the inflammation (toxin production, disruption of epithelial barrier, etc.) was not studied. The intestinal inflammation was evaluated by the changes of cytokines and intestinal histology.

To conclude whether an animal model is successful, DAI scores and tissue damage indices are frequently used by the majority of researchers. In addition, some immune parameters (such as $\operatorname{IgA}$ and $\operatorname{IgG}$ ), cytokines (such as TNF- $\alpha$ and IL-1 $\alpha)(9,12)$ and biochemical indices (such as MPO, malondialdehyde, and superoxide dismutase) are also used. In this study, we found that the body weight of the model groups was reduced to its lowest value and the DAI scores were the highest on the $8^{\text {th }}$ day, and there were also significant pathological changes in the duodenum. MPO is a haeme protease, and its concentration in tissues may indicate the number of inflammatory cells. IL- $1 \alpha$ and TNF- $\alpha$ are proinflammatory cytokines secreted by helper $\mathrm{T}$ cells, dendritic cells, and other cells during inflammation, which can induce the occurrence and expansion of inflammation. In this study, we found that the levels of IL- $1 \alpha$, TNF- $\alpha$, and MPO in the peripheral blood of the model groups were the highest on day 8 , and there were significant differences in these levels compared with the control group $(\mathrm{P}<0.05)$. The results demonstrated that ETEC K88 C83912 can induce an inflammatory reaction in mouse duodenum, which is most severe on day 8. All these data are useful for basic research on immune mechanisms regulating intestinal barrier function.

Conflict of Interests Statement: The authors declare that they have no conflict of interests regarding the publication of this article.

Financial Disclosure Statement: This work was supported by the China Natural Science Foundation (No. 31372413).

Animal Rights Statement: The protocol was approved by the Committee on the Ethics of Animal Experiments of the Jilin Agricultural University, China.

\section{References}

1. Cristina W.C., Donal O.T., Naomi S.T., Donald P.K., Hong L.: Are rabbits a suitable model to study sheep-associated malignant 
catarrhal fever in susceptible hosts? Vet Microbiol 2013, 163, 358-363.

2. Chen Q., Shen H., Yu S.Y.: Experimental study on the pathogenicity of enterotoxigenic Escherichia coli in guinea pig. Chinese J Zoonoses 2000, 16, 48-51.

3. Fairbrother J.M., Nadeau E., Gyles C.L.: Escherichia coli in postweaning diarrhea in pigs: an update on bacterial types, pathogenesis, and prevention strategies. Anim Health Res Rev $2005,6,17-39$

4. Julian R.M., Takuichi S., Andrew J.W., Tracey A.M., John C.F., Sarah J.H., William G.W.: Design and evalution of useful bacterium-specific PCR primers that amplify genes coding for bacterial 16S rRNA. Appl Environ Microb 1998, 64, 795-799.

5. Kerry K., Cooper J., Glenn S.: Virulence of Clostridium perfringens in an experimental model of poultry necrotic enteritis. Vet Microbiol 2010, 142, 323-328.

6. Kwang-il J., Malabi M., Venkatesan J., Shoshana B., Saul T.: Evaluation of virulent and live Shigella sonnei vaccine candidates in a gnotobiotic piglet model. Vaccine 2013, 31, 4039-4046.

7. Liu Y., Sui L.H., Zeng L.: Establishment of a mouse model of Escherichia coli $\mathrm{O} 127$ infection and real-time PCR detection of TGF- $\beta 1$ expression. Acta Lab Anim Sci Sin 2013, 21, 13-16.

8. Mizoguchi A.: Animal models of inflammatory bowel disease. Prog Mol Biol Transl Sci 2012, 105, 263-320.
9. Mohammad F.R., Labis B., Boutigue M.M.: Catestatin decreases macrophage function in two mouse models of experimental colitis. Biochem Pharmacol 2014, 89, 386-398.

10. Nemec A., Pavlica Z., Crossley D.A., Zdovc I., Erzen D., Petelin M., Nemec M., Šentjurc M.: Single oral inoculation with Escherichia coli (ATCC 25922) stimulates generalised production of nitric oxide in mice. Acta Vet Hung 2009, 1, 127-138.

11. Randolph M.M., Allen K.P., Fleckenstein J.M.: Importance of heat-labile enterotoxin in colonization of the adult mouse small intestine by human enterotoxigenic Escherichia coli strains. Infect Immun 2006, 74, 869-875.

12. Sha T., Igaki K., Yamasaki M., Watanabe T., Tsuchimori N.: Establishment and validation of a new semi-chronic dextran sulfate sodium-induced model of colitis in mice. Int Immunopharmacol 2013, 15, 23-29.

13. Vassilis V., Giorgos B., George K.: Experimental colitis models: Insights into the pathogenesis of inflammatory bowel disease and translational issues. Eur J Pharmacol 2015, 759, 253-264.

14. Yu H., Wang L., He J.: Construction of Lactobacillus pentose expressing porcine lactoferrin and evaluation of the immune effect in mice against $E$. coli K88 challenge. Chinese J Prevent Vet Med 2014, 36, 889-893. 